\title{
Selection for tetracycline-resistant Chlamydia suis in treated pigs
}

Borel, Nicole ; Regenscheit, N ; Di Francesco, A ; Donati, M ; Markov, J ; Masserey, Y ; Pospischil, A

\begin{abstract}
The aim of this study was to investigate Chlamydia suis in a pig farm with an outbreak of conjunctivitis and diarrhea. Eye swabs and pooled fecal samples were investigated for the presence of C. suis by real-time PCR and ArrayTube microarray. Samples positive for C. suis by ArrayTube microarray assay were further tested for the presence of the tet $(\mathrm{C})$ resistance gene by PCR. In the first examination, C. suis was identified in 12 six-week-old pigs showing conjunctivitis. Of these, the tet(C) gene-coding region was amplified in one pooled fecal sample and one eye swab, respectively. After oral treatment with tetracycline, clinical symptoms disappeared. Subsequently, all eye swabs investigated from 10 healthy pigs were positive for C. suis and the tet $(\mathrm{C})$ gene-coding region. The present study reports rapid selection for tetracycline-resistant C. suis after antibiotic treatment.
\end{abstract}

DOI: https://doi.org/10.1016/j.vetmic.2011.10.011

Posted at the Zurich Open Repository and Archive, University of Zurich

ZORA URL: https://doi.org/10.5167/uzh-54309

Journal Article

Accepted Version

Originally published at:

Borel, Nicole; Regenscheit, N; Di Francesco, A; Donati, M; Markov, J; Masserey, Y; Pospischil, A (2012). Selection for tetracycline-resistant Chlamydia suis in treated pigs. Veterinary Microbiology, 156(1-2):143146.

DOI: https://doi.org/10.1016/j.vetmic.2011.10.011 


\section{Accepted Manuscript}

Title: Selection for Tetracycline-Resistant Chlamydia suis in Treated Pigs

Authors: Nicole Borel, Nadine Regenscheit, Antonietta Di

Francesco, Manuela Donati, Jenny Markov, Yvonne Masserey, Andreas Pospischil

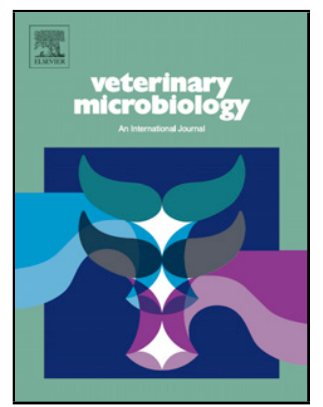

PII: S0378-1135(11)00554-2

DOI: doi:10.1016/j.vetmic.2011.10.011

Reference: VETMIC 5497

To appear in: $\quad$ VETMIC

Received date: 25-7-2011

Revised date: $\quad 4-10-2011$

Accepted date: $\quad$ 7-10-2011

Please cite this article as: Borel, N., Regenscheit, N., Di Francesco, A., Donati, M., Markov, J., Masserey, Y., Pospischil, A., Selection for Tetracycline-Resistant Chlamydia suis in Treated Pigs, Veterinary Microbiology (2010), doi:10.1016/j.vetmic.2011.10.011

This is a PDF file of an unedited manuscript that has been accepted for publication. As a service to our customers we are providing this early version of the manuscript. The manuscript will undergo copyediting, typesetting, and review of the resulting proof before it is published in its final form. Please note that during the production process errors may be discovered which could affect the content, and all legal disclaimers that apply to the journal pertain. 
1 Selection for Tetracycline-Resistant Chlamydia suis in Treated Pigs

2

3 Nicole Borel $^{1 \mathrm{a}}$, Nadine Regenscheit ${ }^{1 \mathrm{a}}$, Antonietta Di Francesco $^{2}$, Manuela Donati ${ }^{3}$,

4 Jenny Markov ${ }^{4}$, Yvonne Masserey $^{4}$, Andreas Pospischil $^{1}$

5

61 Institute for Veterinary Pathology, University of Zurich, Vetsuisse Faculty,

7 Winterthurerstrasse 268, $\mathrm{CH}-8057$ Zurich, Switzerland

82 Department of Veterinary Medical Sciences, University of Bologna, Via Tolara di

9 Sopra, 50, 40064 Ozzano Emilia (BO), Italy

103 Section of Microbiology DESOS, Policlinico S. Orsola, University of Bologna, Via

11 Massarenti 9, 40138 Bologna, Italy

124 SUISAG pig health service (PHS), Winterthurerstrasse 260, $\mathrm{CH}-8057$ Zurich,

13 Switzerland

14

15

16 aThese two authors contributed equally to this study

17

18 Corresponding author:

19 Nicole Borel, DVM, FVH, Dipl. ECVP

20 Institute of Veterinary Pathology, Vetsuisse Faculty

21 University of Zurich, Winterthurerstrasse 268

$22 \mathrm{CH}-8057$ Zurich, Switzerland

23 Tel.: +41-44-635-8576

24 Fax.: +41-44-635-8934

25 Email: n.borel@access.uzh.ch 


\section{$1 \quad$ Abstract}

2 The aim of this study was to investigate Chlamydia suis in a pig farm with an

3 outbreak of conjunctivitis and diarrhea. Eye swabs and pooled fecal samples were

4 investigated for the presence of $C$. suis by real-time PCR and ArrayTube microarray.

5 Samples positive for $C$. suis by ArrayTube microarray assay were further tested for

6 the presence of the tet(C) resistance gene by PCR. In the first examination, $C$. suis

7 was identified in 12 six-week old pigs showing conjunctivitis. Of these, the tet(C)

8 gene-coding region was amplified in one pooled fecal sample and one eye swab,

9 respectively. After oral treatment with Tetracycline, clinical symptoms disappeared.

10 Subsequently, all eye swabs investigated from ten healthy pigs were positive for $C$.

11 suis and the tet(C) gene-coding region. The present study reports rapid selection for

12 Tetracycline-resistant $C$. suis after antibiotic treatment.

14 Key words

15 Chlamydia suis, conjunctivitis, diarrhea, selection, swine, Tetracyline-resistance

17 Introduction

Chlamydia (C.) suis is associated with respiratory disease, diarrhea and conjunctivitis in pigs (Pospischil et al., 2010). Chlamydiae are commonly found in the intestine of pigs mostly associated with subclinical infections (Nietfeld et al., 1997). However, C. suis caused enteritis when inoculated in gnotobiotic piglets (Rogers and Andersen, 1996; Guscetti et al., 2009) or caused histologically intestinal lesions in asymptomatic young weanling pigs (Rogers and Andresen, 2000). Tetracycline-resistant C. suis strains have been described in the US (Lenart et al., 2001) and in Italy (Di Francesco et al., 2008). In vitro, chlamydial inclusions contain aberrant forms when cultured in addition of tetracycline indicating the induction of persistence by the addition of 
1 antibiotics (Lenart et al., 2001). In vivo, aberrant chlamydial developmental forms in

2 the gastrointestinal tract of pigs spontaneously and experimentally infected with $C$.

3 suis have been shown (Pospischil et al., 2009). In the latter study, group A consisted

4 of conventionally reared pigs from a farm in the US and was used for experimental

5 infection with Salmonella typhimurium. These pigs were by chance detected to be

6 naturally infected with $C$. suis, but unavailability of fresh tissue material prevented the

7 amplification of the Tetracyline $(\operatorname{tet}(\mathrm{C}))$ resistance gene (data not shown). Group B

8 consisted of gnotobiotic piglets experimentally infected with the C. suis strain S45/6

9 which is known to lack the tet(C) gene-coding region. Interestingly, this strain was

10 harvested originally in the late 1960s from feces of an asymptomatic pig in Austria

11 indicating the presence of non-resistant $C$. suis strains in Europe at that time.

12 Tetracyclines are widely used in veterinary medicine because they are relatively

13 inexpensive and have a broadspectrum activity. To date, selection of Tetracyline-

14 resistance in C. suis in pigs after antibiotic treatment is not reported.

Material and Methods

17 In April 2010, a Swiss breeding pig farm consisting of approximately 400 animals had problems of wasting, decreased body weight, diarrhea and conjunctivitis in weaned pigs. Fertility parameters in breeding sows were normal. A vaccination protocol for Lawsonia intracellularis, porcine Circovirus, porcine Parvovirus, Escherichia coli,

21 Clostridium sp. and Erysipelas was performed in the herd. Deworming and iron prophylaxis was regularly followed and the hygiene management of the herd was good. Eye swabs of 12 six-week old pigs showing conjunctivitis (conjunctival chemosis and reddening, ocular discharge) and pooled fecal samples $(n=3)$ of their 25 housing compartments were taken for further examination. Subsequently, all pigs of 26 the farm were treated orally with tetracycline for 3 weeks. In August 2010, no clinical 
1 symptoms were recorded in the farm. Control eye swabs were taken from ten healthy

2 pigs.

3 DNA of all samples (eye swabs: $n=22$; pooled fecal samples: $n=3$ ) was extracted 4 using the DNeasy Blood and Tissue Kit (QIAGEN, Hilden, Germany) according to the 5 manufacturer's instructions. All samples were examined on an $A B I 7500$ instrument

6 (Applied Biosystems, Foster City, CA, USA) using the 23S rRNA gene-based

7 Chlamydiaceae family-specific real-time PCR as described previously (Ehricht et al.,

8 2006). Briefly, this method includes primers Ch23S-F (5'-

9 CTGAAACCAGTAGCTTATAAG CGGT-3'), Ch23S-R (5'ACCTCGCCGTTTAACTTAACTCC-3'), and probe Ch23S-p (FAM-CTCATCA TGCAAAAGGCACGCCG-TAMRA) and an internal amplification control consisting of primers EGFP-1-F (5'-GACCACTACCAGCAGAACAC-3'), EGFP-10-R (3'CTTGTACAGCTCGTCCATGC-5') and probe EGFP-HEX (HEXAGCACCCAGTCCGCCCTGAGCA-BHQ1). A 111-bp product specific for members of the family Chlamydiaceae is produced as well as a 177-bp product for the internal amplification control. A cycle threshold (Ct value) of $<38.00$ was considered as positive, and all samples were tested at least in duplicate. The samples with positive Ct values were examined using the species-specific 23S ArrayTube (AT) microarray (Alere, Jena, Germany) assay (Borel et al., 2008). Samples positive for C. suis by AT microarray assay were further tested for for the presence of the tet(C) resistance gene by a PCR assay amplifying a 525 base pair product of the tet(C) gene-coding region. The reaction was performed according to Dugan and others (2004), using following forward and reverse oligonucleotide primers: CS43 5'AGCACTGTCCGACCGCTTTG-3' and CS47 5'-TCCTCGCCGAAAATGACCC-3'. The S45 tetracycline-sensitive and the MS08 tetracycline-resistant C. suis strains were used as negative and positive control, respectively. The amplicons were 
1 purified using a commercially available kit (QIAquick PCR Purification Kit, QIAGEN,

2 Hilden, Germany), sequenced (Bio-Fab Research, Rome, Italy) and identified

3 through BLAST search (www.ncbi.nlm.nih.gov/blast/).

5 Results and Discussion

6 Real-time PCR for Chlamydiaceae was positive in all 12 eye swabs and the pooled

7 fecal samples (n=3) in April 2010. By AT microarray, C. suis was identified in all samples (Figure 1). Of these, the tet(C) gene-coding region was amplified in one pooled fecal sample and one eye swab, respectively. Amplicons carried an identical

10 nucleotide sequence that showed $100 \%$ homology with those of the structural gene

11 tet(C) described by Dugan and others (2004) (Gene Bank Accession AY428551).

12 Other infective agents causing conjunctivitis and diarrhea were excluded by

13 laboratory investigations (data not shown). In August 2010, no clinical symptoms

14 were recorded in the farm. All eye swabs $(n=10)$ collected from healthy pigs were

15 positive for $C$. suis and the tet $(\mathrm{C})$ gene-coding region.

16 Chlamydiae were reported as a cause of conjunctivitis in pigs in Nebraska in 1993

17 (Rogers et al., 1993). As in this study, weanling pigs were affected and they responded well to oxytetracycline treatment. At that time, no clear classification of the involved chlamydial species was performed. C. suis was found in a study in pigs with conjunctivitis but also in healthy controls from Switzerland and Germany (Becker et al., 2007). A concurrent outbreak of chlamydial disease and postweaning multisystemic wasting syndrome was recently described in weaned piglets in a pig production farm in Estonia (Schautteet et al., 2010). C. suis DNA was detected in eye swabs from boars, sows and gilts with conjunctivitis and also in feces of boars and sows. Therapy with doxycycline was not successful and clinical signs persisted. The authors allocated this to either to short treatment period, to prolonged shipment of the 
1 samples, or to the presence of Tetracycline-resistant strains. In both studies (Becker

2 et al., 2007; Schautteet et al., 2010), C. suis-strains were not further investigated for

3 the Tetracycline-resistance gene. In another study (Reinhold et al., 2011), short-term

4 treatment with enrofloxacin or enrofloxacin plus tiamulin was not effective in

5 eliminating subclinical infections with $C$. suis in pigs. In the present study, clinical

6 symptoms disappeared after antibiotic treatment but $C$. suis was not eliminated and

7 strains harboring the $\operatorname{tet}(\mathrm{C})$ resistance gene were positively selected. As recently

8 reviewed by Sandoz and Rockey (2011), the discovery of the tet(C) islands

9 represents the first identification of antibiotic resistance acquired through horizontal

10 gene transfer in any obligate intracellular bacteria. The mechanism of acquisition of

11 the tet $(\mathrm{C})$ islands in C. suis remains speculative. However, acquisition of DNA from

12 other bacteria by Chlamydia that commonly infect the porcine intestinal tract such as

13 C. suis is conceivable. The rapid selection for Tetracycline-resistant C. suis strains

14 after antibiotic treatment in this study was surprising and possibly facilitated by the

15 close contact of pigs in a relatively small farm (400 animals). In vitro experiments

16 have demonstrated the acquisition of Tetracycline resistance by horizontal gene

17 transfer from C. suis to clinical strains of C. trachomatis (Suchland et al., 2009). In

18 vivo, such events are of particular public health concern if the tet(C) resistance gene

19 is transferred from porcine chlamydial strains to human pathogens.

20 C. suis was found in pooled fecal samples of diarrheic pigs in the present study.

21 Prophylactic vaccination against common enteropathogenic agents was performed in

22 this herd and other infectious agents were excluded by laboratory investigations

23 (data not shown). This implies C. suis as the sole agent causing diarrhea in this herd,

24 although mostly subclinical infections have been reported in previous field studies

25 (Nietfeld et al., 1997). It is known that different isolates of C. suis exist with a high 
1 degree of genetic diversity possibly leading to variation in virulence. Further

2 characterization of these chlamydial strains is currently under investigation.

\section{Conclusion}

5 This study resulted in the first description of Tetracycline-resistant C. suis in a Swiss

6 pig farm with conjunctivitis and diarrhea. Excretion of these strains by ocular and

7 fecal route was present before and after antibiotic treatment. After tetracycline

8 treatment, strains harboring the tet(C) gene-coding region were predominantly

9 prevalent indicating selection of these under treatment conditions. The adaptive

10 availability to acquire the tet(C)-resistant gene when exposed to selective pressure

11 could have far-reaching consequences for the emergence of antibiotic resistance in

12 chlamydiae and could pose a potential threat for the food safety. Further

13 investigations in other European countries to detect tet(C)-resistant strains are

14 needed to increase awareness and caution when treating pigs with antibiotics.

16 Conflict of interest statement

17 All authors declare there is no financial or personal relationships with other people or organizations that could have inappropriately influenced their work.

Acknowledgments

21 We are grateful to Carmen Kaiser of the laboratory of the Institute of Veterinary

22 Pathology, University of Zurich for technical help.

References

Becker, A., Lutz-Wohlgroth, L., Brugnera, E., Lu, Z.H., Zimmermann, D.R., Grimm,

F., Grosse Beilage, E., Kaps, S., Spiess, B., Pospischil, A., Vaughan, L., 2007. 
1 Intensively kept pigs pre-disposed to chlamydial associated conjunctivitis. J. Vet.

2 Med. A. Physiol. Pathol. Clin. Med. 54, 307-313.

3

4 Borel, N., Kempf, E., Hotzel, H., Schubert, E., Torgerson, P., Slickers, P., Ehricht, R.,

5 Tasara, T., Pospischil, A., Sachse, K., 2008. Direct identification of chlamydiae from

6 clinical samples using a DNA microarray assay - A validation study. Mol. Cell.

7 Probes. 22, 55-64.

8

9 Di Francesco, A., Donati, M., Rossi, M., Pignanelli, S., Shurdhi, A., Baldelli, R.,

10 Cevenini, R., 2008. Tetracycline-resistant Chlamydia suis isolates in Italy. Vet. Rec.

$11 \quad 163,251-252$.

12

13 Dugan, J., Rockey, D.D., Jones, L., Andersen, A.A., 2004. Tetracycline resistance in

Chlamydia suis mediated by genomic islands inserted into the chlamydial inv-like gene. Antimicrob. Agents. Chemother. 48, 3989-3995.

16

17 Ehricht, R., Slickers, P., Goellner, S., Hotzel, H., Sachse, K., 2006. Optimized DNA microarray assay allows detection and genotyping of single PCR-amplifiable target copies. Mol. Cell. Probes. 20, 60-63.

21 Guscetti, F., Schiller, I., Sydler, T., Heinen, E., Pospischil, A., 2009. Experimental 22 enteric infection of gnotobiotic piglets with Chlamydia suis strain S45. Vet. Microbiol. $135,157-168$.

Lenart, J., Andersen, A.A., Rockey, D.D., 2001. Growth and development of

26 Tetracycline-resistant Chlamydia suis. Antimicrob. Agents. Chemother. 45, 2198- 
3 Nietfeld, J.C., Leslie-Steen, P.L., Zeman, D.H., Nelson, D., 1997. Prevalence of

4 intestinal chlamydial infection in pigs in the midwest, as determined by

5 immunoperoxidase staining. Am. J. Vet. Res. 58, 260-264.

6

7 Pospischil, A., Borel, N., Andersen, A.A., 2010. Chlamydia. In: Gyles, C.L., Prescott,

8 J.F., Songer, J.G., Thoen, C.O. (Eds.), Pathogenesis of bacterial infections in

9 animals. Blackwell Publishing, pp. 575-87.

11 Pospischil, A., Borel, N., Chowdhury, E.H., Guscetti, F., 2009. Aberrant chlamydial

12 developmental forms in the gastrointestinal tracts of pigs spontaneously and

13 experimentally infected with Chlamydia suis. Vet. Microbiol. 135, 147-56.

15 Reinhold, P., Liebler-Tenorio, E., Sattler, S., Sachse, K., 2011. Recurrence of

16 Chlamydia suis infection in pigs after short-term antimicrobial treatment. Vet. J. 187, $17 \quad 405-407$.

19 Rogers, D.G., Andersen, A.A., 1996. Intestinal lesions caused by two swine

20 chlamydial isolates in gnotobiotic piglets. J. Vet. Diagn. Invest. 8, 433-440.

21 Rogers, D.G., Andersen, A.A., 2000. Intestinal lesions caused by a strain of

22 Chlamydia suis in weanling pigs infected at 21 days of age. J. Vet. Diagn. Invest. 12, 23 233-239. 
1 Rogers, D.G., Andersen, A.A., Hogg, A., Nielsen, D.L., Huebert, M.A., 1993.

2 Conjunctivitis and keratoconjunctivitis associated with chlamydiae in swine. J. Am.

3 Vet. Med. Assoc. 203, 1321-1323.

4 Sandoz, K.M., Rockey, D.D., 2010. Antibiotic resistance in Chlamydiae. Future 5 Microbiol. 5, 1427-1442.

6 Schautteet, K., Beeckman, D.S.A., Delava, P., Vanrompay, D., 2010. Possible

7 pathogenic interplay between Chlamydia suis, Chlamydophila abortus and PCV-2 on

8 a pig production farm. Vet Rec. 166, 329-333.

9 Suchland, R.J., Sandoz, K.M., Jeffrey, B.M., Stamm, W.E., Rockey, D.D., 2009.

10 Horizontal transfer of Tetracycline resistance among Chlamydia spp. in vitro.

11 Antimicrob. Agents. Chemother. 53, 4604-4611.

14 Figure captions

15 Fig. 1. DNA-based ArrayTube Microarray species identification assay of eye swab

16 from a pig positive for Chlamydiaceae by real-time PCR (mean Ct value 24.77).

17 Barplot showing specific signals for genus Chlamydia (1) and species Chlamydia suis

18 (2). Red bars represent the perfect-match probe signal, blue bars show the

19 hybridization signal with the probe carrying one or two mismatches at the 3 '-end. 


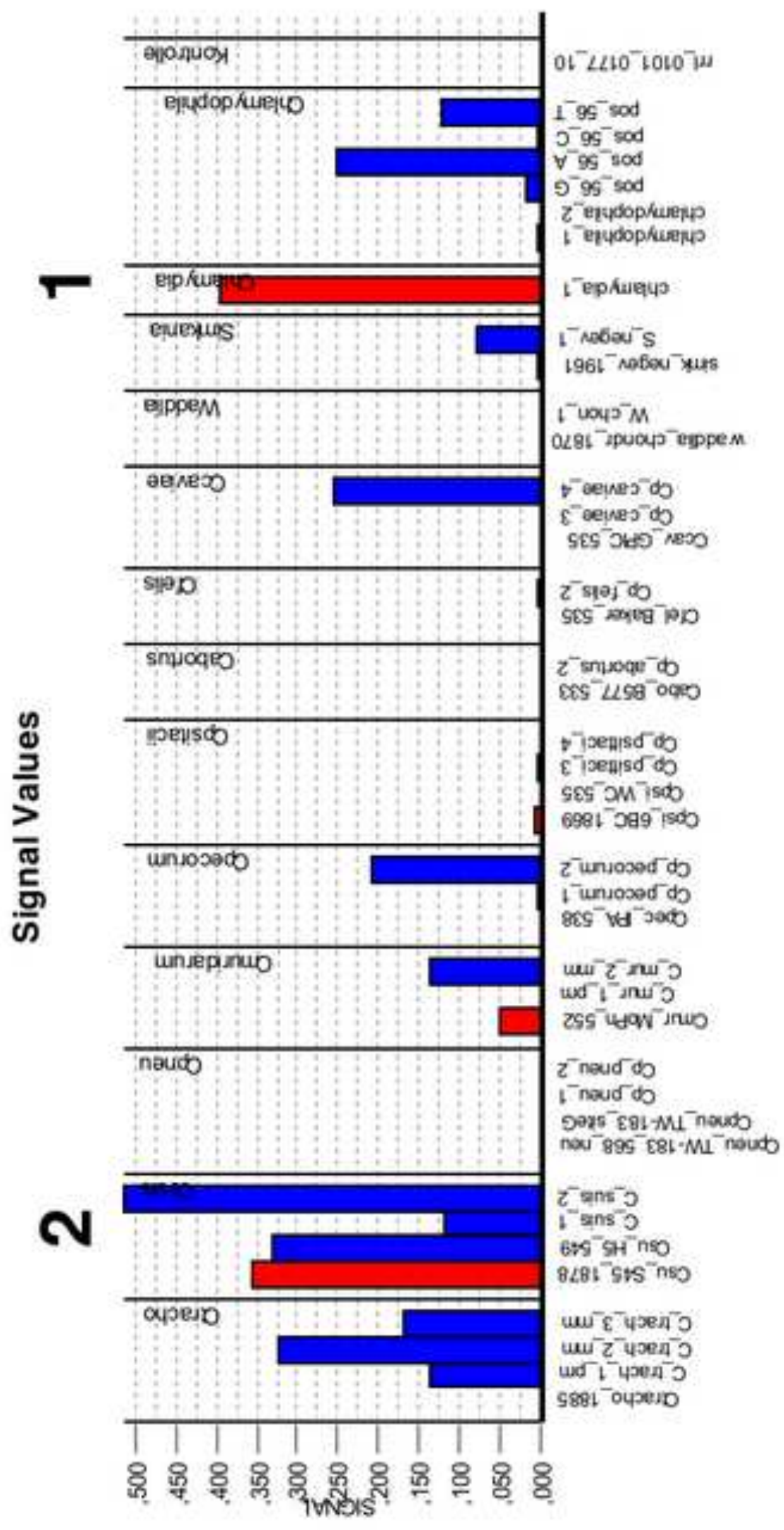

$\frac{w}{8}$ 